\title{
Propiedades psicométricas de la Escala de Satisfacción Familiar de Olson y Wilson en estudiantes universitarios
}

Psychometric properties of the Olson and Wilson's Family

Satisfaction Scale in university students

Walter L. Arias Gallegos

Renzo Rivera

Patricia Laurie Heresi

Karla D. Ceballos Canaza

Universidad Católica San Pablo, Perú

Correspondencia:warias@ucsp.edu.pe

Recibido: 21-03-2019. Revisado: 22-05-2019. Aceptado: 13-09-2019

Citarlo como: Arias, W. L., Rivera, R., Laurie, P., \& Ceballos, K. (2019). Propiedades psicométricas de la Escala de Satisfacción Familiar de Olson y Wilson en estudiantes universitarios. Perspectiva de Familia, 4, pp. 47-66.

\section{Resumen}

En este artículo se analizan las propiedades psicométricas de la Escala de Satisfacción Familiar de Olson y Wilson (1982) en una muestra de estudiantes universitarios de la ciudad de Arequipa. Para lo cual se aplicó dicho instrumento a 170 estudiantes de una universidad privada, que tienen una edad entre 17 y 40 años, siendo la mayoría de ellos de sexo femenino (6o \%) y de estado civil soltero (93\%). Los resultados indican que la Escala de Satisfacción Familiar tiene óptimos niveles de validez y confiabilidad, pero estos valores son superiores cuando se trabaja con un modelo unidimensional, en lugar de uno bifactorial, como propuso originalmente Olson.

Palabras clave: Satisfacción familiar, validez, confiabilidad, estudiantes universitarios. 


\begin{abstract}
In this article, we analyze the psychometric properties of the Family Satisfaction Scale (Olson \& Wilson, 1982) in a simple of university students from Arequipa City. In order to get our aim, we applied the scale to 170 university students from a private university located in Arequipa, aged 17 to 40 years old, being the most of them female $(60 \%)$ and single (93\%). The results indicate that the Family Satisfaction Scale has good levels validity and reliability, but they are higher when we consider a unidimensional model, instead of a two-factor model, such as it was proposed originally by Olson.
\end{abstract}

Key words: Family satisfaction, validity, reliability, university students.

La familia ha sido considerada como la célula fundamental de la sociedad y, dada su importancia, ha merecido un sinnúmero de investigaciones en todo el mundo (Arias, 2013); de modo tal que se cuenta con revistas especializadas en temas de familia (Family Process, Journal of Marriage and Family, Journal of Family Studies, Journal of Family Psychology, Journal of Family Violence, Journal of Family Therapy, Journal of Child and Family Studies, Journal of Couple and Family Psychology, European Journal of Family Business, etc.) e instituciones, sociedades y organismos internacionales comprometidos con su desarrollo y promoción.

También ha sido objeto de estudio de diversas disciplinas, tales como el derecho, la sociología, la historia, la medicina, la biología, la educación y la psicología. En ese sentido, la psicología no ha sido ajena a la relevancia que tiene la familia en la formación y el desarrollo psicológico del niño o del bienestar de la familia en tanto matrimonio y colectivo de personas que hacen vida común, pues la American Psychological Association (APA) cuenta con dos divisiones orientadas al tema de la familia. La primera es la división 37 Child and Family Policy and Practice y la división 43 Couple and Family Psychology.

En el Perú existen diversos estudios sobre la familia realizados por psicólogos, y que se concentran básicamente en tres ciudades: Lima, Arequipa y Trujillo. Estos estudios comprenden una amplia diversidad de temas que podemos agrupar en las siguientes categorías: violencia familiar (Arias, Galagarza, Rivera \& Ceballos, 2017; Delgado, 2016; Castro \& Rivera, 2015; Castro, Cerellino \& Rivera, 2017; Miljánovich et al., 2010; Miljánovich et al., 2013; Minetto, 2013), el impacto de la familia en la educación (Arias, Quispe \& Ceballos, 2016; Sotil, 2002), familia y salud mental (Araujo, 2005; Capa, Vallejos \& Cárdenas, 2010; Galagarza \& Arias, 2017; Mallma, 2016; Mayorga \& Niquen, 2010; Pérez, 2016; Rivera \& Cahuana, 2016; Rosas, 2014; Tirado 
et al., 2008; Yucra, 2016), familia y bienestar (Alarcón, 2014; Arias, Masías, Salas, Yépez \& Justo, 2014; Cárdenas, 2016; Caycho, Contreras \& Merino, 2016), estructura o composición familiar (Chuquimajo, 2017; Laguna \& Rodríguez, 2008; Prado \& Del Águila, 2004; Reusche, 1995; Silva \& Argote, 2007; Villarreal-Zegarra, 2015; Villarreal-Zegarra \& Paz-Jesús, 2017), familia, trabajo y economía (Arias \& Ceballos, 2016; Castro et al., 2013; Castro, Rivera \& Seperak, 2017; Prado \& Del Águila, 2010), estilos de crianza y comunicación con los padres (Araujo, 2007, 2008; Muñoz, 2016; Reusche, 1999; Sobrino, 2008), satisfacción marital (Rebaza \& Julca, 2009), clima y/o ambiente familiar (Cruz, 2013; Matalinares et al., 2010; Oruna, 2016) y cualidades psicométricas de pruebas que evalúan constructos relativos a la familia (Arias et al., 2013; Castilla, Caycho, Shimabukuro \& Valdivia, 2014; Copez-Lonzoy, Villarreal-Zegarra \& Paz-Jesús, 2016; Villarreal-Zegarra, Paz-Jesús, Copez-Lonzoy \& Costa-Ball, 2017; Dominguez \& Alarcón, 2017; Dominguez, Aravena, Ramírez \& Yauri, 2013; Matalinares, Raymundo \& Baca, 2014; Merino \& Arndt, 2004; Manrique, Ghesquière \& Van Leeuwen, 2014; Merino, Díaz \& Cohen, 2003; Merino, Díaz \& DeRoma, 2004).

De hecho, en el Perú hay dos institutos orientados a la familia. Uno de ellos es el Instituto de la Familia de la Universidad Femenina del Sagrado Corazón (UNIFÉ), que tiene una orientación marcadamente jurídica y que existe desde el año 2001, previa creación de la primera Maestría en Derecho Civil con Mención en Familia que se dictó en el país. Asimismo, desde el año 2012 edita la revista Persona y Familia, a razón de un número por año (Vidal, 2014). La otra institución a la que nos referimos es el Instituto para el Matrimonio y la Familia de la Universidad Católica San Pablo, de Arequipa, que en el año 1998 inició una labor de orientación psicológica y asesoría jurídica a la población sobre temas de familia, tarea que continuó hasta el 2014, año en que empezó a tener una orientación más académica con énfasis en la investigación. A su vez, desde el año 2016 edita la revista Perspectiva de Familia como órgano de difusión.

Arequipa ha sido una ciudad con marcado interés por la familia; en ese sentido, la psicología ha aportado con diversas publicaciones. Belén Salvatierra (1992, 1993, 1997), por ejemplo, ha publicado diversos libros sobre orientación familiar y matrimonial desde la década de los 9o. Asimismo, en la Universidad Católica de Santa María se han llevado a cabo estudios sobre el impacto de la estructura familiar, en aspectos tales como las actitudes hacia el matrimonio (Silva \& Argote, 2007), la inteligencia emocional de los hijos (Oporto \& Zanabria, 2006) o la resiliencia en niños de edad preescolar (Laguna \& Rodríguez, 2008).

En la Universidad Católica San Pablo, desde la Escuela Profesional de Psicología, se han realizado algunos estudios sobre la familia, como el juicio moral (Murillo, 
2015) y los motivos para contraer matrimonio en casados y divorciados (Seperak, 2016). También se han hecho algunos aportes desde un enfoque sistémico familiar a nivel teórico y empírico sobre violencia transgeneracional y funcionamiento familiar en estudiantes de nivel universitario (Arias, 2012, 2013; Galagarza \& Arias, 2017). Desde el Instituto para el Matrimonio y la Familia, se han efectuado diversas investigaciones sobre integración familiar (Arias et al., 2013; Arias et al., 2014; Arias \& Ceballos, 2016; Castro et al., 2013), violencia contra la mujer (Arias et al., 2017; Castro \& Rivera, 2015; Castro, Cerellino et al., 2017), estructura familiar y bienestar económico (Castro et al., 2013; Castro, Riesco \& Arela, 2016; Castro, Rivera et al., 2017; Pliego \& Castro, 2015; Riesco \& Arela, 2015) y el impacto de variables familiares en la conducta y la salud mental de los adolescentes (Rivera \& Cahuana, 2016; Rivera, Arias \& Cahuana, en revisión).

A nivel psicométrico se ha creado el Inventario de Integración Familiar (Arias et al., 2013), que sería el primer instrumento de factoría peruana que evalúa a la familia. En relación con esto, son pocos los instrumentos validados en el país que valoren aspectos propios de la familia, aunque se pueden destacar: el Inventario de Percepción de Conducta Parental (Merino et al., 2003; Merino et al., 2004), la Escala de Estilos de Crianza de Steinberg (Merino \& Arndt, 2004), la Escala de Estilos Parentales (Manrique et al., 2014; Matalinares et al., 2014), la Escala de Calidad de Interacción Familiar (Dominguez et al., 2013; Dominguez \& Alarcón, 2017) y la Escala APGAR-familiar (Castilla et al., 2014). Sin embargo, una escala ampliamente utilizada en el Perú ha sido el FACES (Family Adaptability and Cohesion Evaluation Scale) y sus diversas versiones, que incluyen la Escala de Satisfacción Familiar de Olson y Wilson (1982).

Cabe señalar que el fundamento teórico de este instrumento reposa en el modelo circumplejo, que se basa en una concepción sistémica de la familia (Olson, 1986, 2000; Olson, Russell \& Sprenkle, 1989). En ese sentido, aunque el modelo sistémico ha sido difundido en el Perú a través de diversos artículos de revisión (Arias, 2012; Sobrino, 1999; Villarreal-Zegarra \& Paz-Jesús, 2015), solo recientemente se han realizado estudios empíricos tomando como fundamento epistemológico a la teoría familiar sistémica (Arias et al., 2017; Galagarza \& Arias, 2017; Villarreal-Zegarra, 2015; Villarreal-Zegarra \& Paz-Jesús, 2017). No obstante, esta escasez de estudios desde un enfoque sistémico es común en los países de América Latina (García \& Schneider, 2017).

Ahora bien, volviendo a la Escala de Satisfacción Familiar de Olson y Wilson, su uso en el Perú ha sido bastante extendido. Reusche $(1995,1999)$ realizó la primera adaptación de la escala y publicó algunas investigaciones en escolares de edad ado- 
lescente. Ferreira (2003) la aplicó a menores en situación de abandono moral, para valorar sus relaciones con la autoestima, y Sobrino (2008) indicó que la satisfacción familiar se relaciona con los estilos de comunicación entre padres e hijos. Mayorga y Ñiquen (2010) encontraron que la satisfacción familiar está relacionada inversamente con la expresión de hostilidad en adolescentes que presentan conductas antisociales, aunque la correlación fue muy baja, pero significativa. Capa et al. (2010) encontraron que, tras su aplicación en adolescentes de zonas urbano-marginales de Lima, la satisfacción familiar tiene poder predictivo para explicar la prevalencia del consumo de sustancias psicoactivas, de modo que, si existe disfuncionalidad familiar, el consumo de alcohol y tabaco aumenta tres veces.

Más recientemente, Villarreal-Zegarra (2015) ha reportado que, en los estudiantes de quinto de secundaria del Callao, la composición familiar se relaciona con cierto grado de cohesión entre sus miembros. Yucra (2016) ha encontrado correlaciones negativas entre la satisfacción familiar y las habilidades sociales, en tanto que Gutara (2016) ha reportado correlaciones inversas, moderadas y significativas entre la satisfacción familiar y el resentimiento en estudiantes de nivel secundario, en Lima. En Arequipa, Galagarza y Arias (2017) han reportado asociaciones significativas entre la alexitimia y la cohesión familiar semirelacionada, siendo más frecuente en mujeres que en varones.

Con respecto a las propiedades psicométricas de la prueba, existen diversas versiones que han dificultado homogeneizar los resultados sobre su validez y confiabilidad, desde formatos basados en adjetivos (Tercero et al., 2013) hasta el modelo de tres factores que ha sido duramente cuestionado, bien porque la escala de comunicación tiene valores bajos de confiabilidad o porque los reactivos que la componen no saturan adecuadamente en el factor correspondiente (Schmidt, Barreyro \& Maglio, 2009). En parte, estos resultados se deben a la gran disparidad de las muestras en que se ha aplicado la Escala de Satisfacción Familiar, que abarca desde mujeres trabajadoras (Aazami, Shamsuddin \& Akmal, 2015) hasta pacientes con traumatismo encefalocraneano (Uderhill, LoBello \& Fine, 2004).

De este modo, la escala FACES comprendía originalmente dos escalas: Adaptabilidad y Cohesión. La primera hace referencia a la flexibilidad del sistema familiar, y comprende aspectos tales como el control, la disciplina, el estilo de negociación, los roles familiares y las reglas que rigen la familia. La Cohesión define el vínculo emocional que une a los miembros de la familia, y comprende las coaliciones, los espacios que ocupan en el sistema familiar y los límites entre los diversos holones o subsistemas familiares (conyugal, parental, fraternal, etc.) (Olson, 200o). 


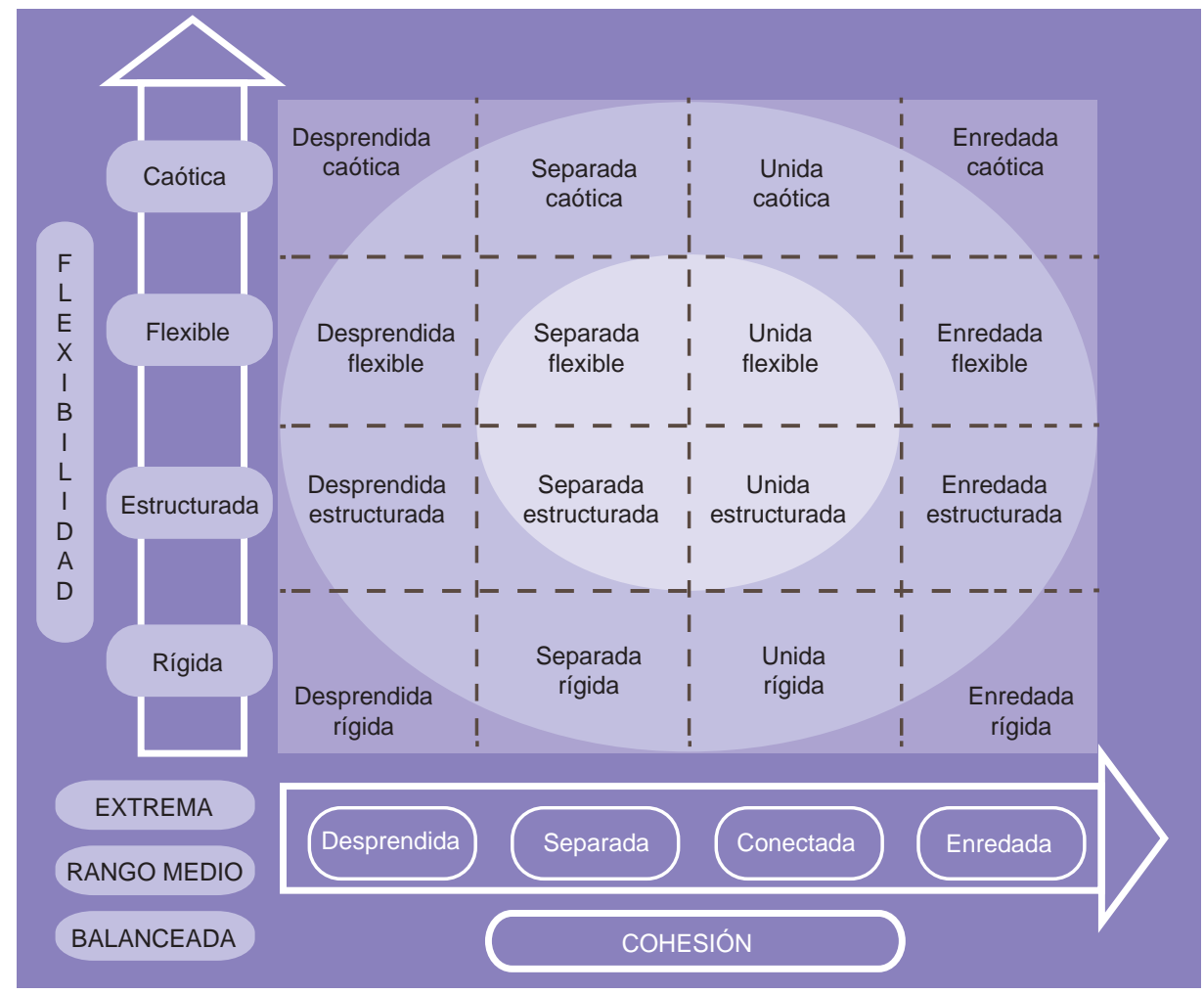

Figura 1.

Modelo Circumplejo de Olson

Asimismo, la Cohesión se da en cuatro niveles que determinan tipos de cohesión: desligada o no relacionada, separada o semirelacionada, conectada o relacionada y amalgamada o aglutinada; mientras que la Adaptabilidad determina otros cuatro niveles: caótica, flexible, estructurada y rígida (Olson, 1993). El cruce de estas dimensiones genera 16 categorías de familias según el grado de interacción que reflejaría su satisfacción familiar (ver Figura 1). Estas 16 categorías se ubican en tres rangos: balanceada (Flexible separada, Flexible conectada, Estructurada separada y Estructurada conectada), medio (Caótica separada, Caótica conectada, Flexible desligada, Flexible amalgamada, Estructurada desligada, Estructurada amalgamada, Rígida separada y Rígida conectada) y extremo (Caótica desligada, Caótica amalgamada, Rígida desligada y Rígida amalgamada), que indican disfuncionalidad o insatisfacción familiar cuando se ubican en un rango extremo (Olson, Portner \& Lavee, 1985). 
Una versión breve del FACES, como la Escala de Satisfacción Familiar, mantiene sus constructos y dimensiones teóricas, pero solo contiene 14 ítems (Olson \& Wilson, 1982), prescindiendo así de la dimensión de Comunicación, que ha sido introducida en versiones más recientes del FACES (Olson et al., 1985; Olson et al., 1989), pero que fue duramente criticada por las razones anteriormente expuestas. Sin embargo, cabe señalar que un estudio con una muestra de 491 estudiantes universitarios peruanos determinó que la escala de comunicación cuenta con adecuados valores de confiabilidad y validez, obtenidas mediante la prueba alfa de Cronbach y análisis factorial confirmatorio, respectivamente (Copez-Lonzoy et al., 2016).

En nuestro país, además, se cuenta con estudios psicométricos del FACES III (Bazo-Alvarez et al., 2016; Villarreal-Zegarra \& Paz-Jesús, 2017), así como de la Escala de Satisfacción Familiar, que han sido aplicadas a estudiantes universitarios y escolares. Capa et al. (2010) han reportado, por ejemplo, que la Escala de Satisfacción Familiar presenta una estructura bifactorial con óptimos indicadores de bondad de ajuste y una confiabilidad de .76 y .70 para las dimensiones de Cohesión y Adaptabilidad, respectivamente; y un coeficiente alfa de Cronbach de .84 para la escala total.

En Arequipa, empero, no se han realizado estudios psicométricos para valorar la validez y la confiabilidad de la Escala de Satisfacción Familiar. Por ello, la finalidad de la presente investigación es analizar las propiedades psicométricas de dicho instrumento en una muestra de estudiantes universitarios de una universidad privada de la ciudad de Arequipa. En consecuencia, se trata de un estudio instrumental (Montero \& León, 2002).

\section{Método}

\section{Participantes}

La muestra estuvo formada por 170 estudiantes de una universidad privada de la ciudad de Arequipa, de los cuales el 6o\% fueron mujeres. Los estudiantes cursaban estudios desde el primero al décimo ciclo de las carreras de Psicología, Ingeniería Industrial, Administración y Derecho. La gran mayoría eran solteros (93.5\%) y el 3.5\% mantenía una relación de matrimonio o de convivencia. Asimismo, tenían una edad promedio de 19.87 años con una desviación estándar de \pm 3.048 dentro de un rango de 17 y 40 años. La muestra fue seleccionada por métodos no probabilísticos a través de la técnica de grupos intactos (Hernández, Fernández \& Baptista, 2006). 


\section{Instrumento}

La Escala de Satisfacción Familiar de Olson y Wilson (1982) consta de 14 ítems, de los cuales, los impares valoran la dimensión de Cohesión, y los pares, la dimensión de Adaptabilidad. Este instrumento es una versión corta de la escala FACES II de Olson. Las alternativas de respuesta tienen cinco niveles en una escala tipo Likert. Los valores se interpretan en función del modelo circunflejo(Olson, 1993, 2000), de modo que los valores extremos indicarían disfuncionalidad familiar, y los valores medios, un equilibrio entre la Cohesión y la Adaptabilidad, que sería indicador de funcionalidad familiar. Validaciones previas hechas en Perú en muestras de adolescentes han reportado que presenta un índice de confiabilidad total de $\alpha=.84$; de $\alpha=.76$, para la dimensión de Cohesión, y de $\alpha=.70$, para la de Adaptabilidad. Asimismo, el análisis factorial exploratorio obtuvo un valor KMO de .902 y dos factores que explicaron el $51.92 \%$ de la varianza total de la prueba. El análisis factorial confirmatorio reveló indicadores adecuados de bondad de ajuste de acuerdo con el modelo propuesto por Olson y Wilson.

\section{Procedimiento}

En primer lugar, se solicitaron los permisos ante las autoridades competentes de la universidad, y se coordinó con los profesores para evaluar a los estudiantes dentro del aula. Al momento de la aplicación del instrumento se explicaron los fines del estudio y se dio el consentimiento informado. Todos los estudiantes participaron voluntariamente y fueron evaluados de manera colectiva con un tiempo aproximado de aplicación de 20 minutos en promedio.

\section{Análisis de datos}

La estructura interna de la Escala de Satisfacción Familiar fue analizada por medio de un Análisis Factorial Confirmatorio (AFC) utilizando el paquete lavaan (Rossel, 2012), el cual fue implementado en el programa estadístico R (R Core Team, 2017). Para su análisis, empleamos el método de Mínimos Cuadrados Diagonalmente Ponderados robusto (DWLS; Muthén, Du Toit \& Spisic, 1997) sobre una base de matrices policóricas (Lee, Poon, \& Bentler, 1995), debido a que los ítems están formulados en una escala tipo Likert, lo que los posiciona como variables ordinales.

Fueron evaluados dos modelos; el primero (M1) fue el de dos factores propuestos originalmente por Olson y Wilson (1982), quienes consideraban que la escala presenta dos factores ortogonales: satisfacción con la vinculación emocional (ítems 1, 3, 5, 7, 9, 11, 13) y satisfacción con la flexibilidad (ítems 2, 4, 6, 8, 10, 12, 14). Así 
mismo, teniendo en cuenta lo reportado por otros investigadores que sugieren que la escala obedece a una estructura unidimensional (Aazami et al., 2015), se generó un modelo (M2) donde todos los ítems son influidos por la misma variable latente. Para la evaluación del modelo se utilizaron diversos índices de bondad de ajuste: la prueba Chi-cuadrado de bondad de ajuste robusto, la cual, si presenta un nivel de significancia menor de .05, nos indica que se debe rechazar el modelo; pero, debido a que el valor $\chi^{2}$ tiende a llevarnos rechazar los modelos propuestos cuando se trabaja con muestras grandes de sujetos (Jöreskog, 1969), las conclusiones se sustentaron en otros criterios, como la razón entre la $\chi^{2}$ sobre sus grados de libertad, que debe ser menor de 3 para indicar que el modelo es adecuado. Además, utilizamos el Î́ndice de Bondad de Ajuste (GFI) y el Î́ndice de Ajuste Comparativo (CFI), los cuales deben tener valores mayores de .9o; la Raíz Cuadrada de la Media de los Residuos (RMR) y la Raíz Cuadrada de Media del Error de Aproximación (RMSEA), que deben ser menores de .o8 (Byrne, 2010). También fueron obtenidas evidencias internas de validez convergente a partir del análisis de la Varianza Promedio Extraída del factor (AVE, Average Variance Extracted) considerando valores por encima de .50 como satisfactorios (Fornell \& Larcker, 1981).

Finalmente, estimamos la fiabilidad del Inventario del sistema de creencias religiosas por medio del coeficiente alfa de Cronbach y el coeficiente Omega de McDonald mediante el software JASP versión o.10.o (JASP Team, 2018).

\section{Resultados}

En la Tabla 1 se puede observar que los ítems tienen una cierta tendencia hacia los puntajes altos. A su vez, 9 ítems presentan índices estandarizados de asimetría (SSI: Standardized Skew Index; Malgady, 2007) de magnitud baja (SSI s $|.25|$ ), lo cual es un indicador de que la mayoría de estos se acercan a presentar una distribución normal. Esto es apoyado por los valores de Z asimetría y Z curtosis (Field, 2009; Hae-Young, 2013), que indican que solo los ítems 1, 9, 11 y 12 no se acercan a tener una distribución normal al tener valores que salen del intervalo [-3.29, 3.29]. 


\section{Tabla 1.}

Estadísticos descriptivos de los ítems

\section{de la Escala de Satisfacción Familiar}

\begin{tabular}{lccccccc}
\hline & Media & $\begin{array}{c}\text { Desv. } \\
\text { estándar }\end{array}$ & $\mathrm{g}_{1}$ & $\mathrm{SSI}$ & $\mathrm{g}_{2}$ & $\begin{array}{c}\mathrm{Z} \\
\text { Asimetría }\end{array}$ & $\begin{array}{c}\mathrm{Z} \\
\text { Curtosis }\end{array}$ \\
\cline { 2 - 7 } Ítem 1 & 3.73 & 1.097 & -.695 & -0.29 & .123 & -3.73 & 0.33 \\
Ítem 2 & 3.69 & 1.015 & -.592 & -0.29 & -.210 & -3.18 & -0.57 \\
Ítem 3 & 3.05 & 1.056 & .015 & 0.01 & -.250 & 0.08 & -0.67 \\
Ítem 4 & 3.39 & 1.142 & -.493 & -0.19 & -.179 & -2.65 & -0.48 \\
Ítem 5 & 3.24 & 1.285 & -.461 & -0.14 & -.706 & -2.47 & -1.91 \\
Ítem 6 & 3.31 & 1.099 & -.443 & -0.18 & -.044 & -2.38 & -0.12 \\
Ítem 7 & 3.17 & 1.151 & -.339 & -0.13 & -.261 & -1.82 & -0.70 \\
Ítem 8 & 3.32 & 1.051 & -.482 & -0.22 & .156 & -2.59 & 0.42 \\
Ítem 9 & 3.77 & 1.157 & -.888 & -0.33 & .320 & -4.77 & 0.86 \\
Ítem 10 & 3.27 & 1.135 & -.525 & -0.20 & .039 & -2.82 & 0.11 \\
Ítem 11 & 3.98 & 1.014 & -.985 & -0.48 & .914 & -5.29 & 2.47 \\
Ítem 12 & 3.86 & 1.116 & -1.163 & -0.47 & 1.257 & -6.25 & 3.39 \\
Ítem 13 & 3.40 & 1.101 & -.497 & -0.20 & -.332 & -2.67 & -0.90 \\
Ítem 14 & 3.42 & 1.076 & -.362 & -0.16 & -.499 & -1.95 & -1.35 \\
\hline
\end{tabular}

Nota: $\mathrm{n}=170$; SSI: Índice Estandarizado de Asimetría; g; : asimetría; g: curtosis

Primeramente, se evaluó el modelo bidimensional, el cual presentó un índice chi cuadrado, indicando así que no tiene un buen ajuste $\chi_{(76)}^{2}=242.172(p<.001) ; \chi^{2} /$ $\mathrm{gl}=3.186$. Aunque los demás índices indican que el modelo es adecuado: $\mathrm{CFI}=.992$; $\mathrm{GFI}=.988 ; \mathrm{AGFI}=.975 ;$ RMSEA [IC 90 \%] = .069 [.05; .087]; SRMR $=.066$. Un punto en contra del modelo bifactorial es que el promedio de varianza explicada es menor de .5 $(\mathrm{AVE}=.466)$. Cabe resaltar que existe una elevada correlación interfactorial $(\phi$ $\geq .90$, ver Tabla 2), lo que sugiere que la Escala de Satisfacción Familiar puede ser unidimensional. 
Tabla 2.

Cargas factoriales y confiabilidad de los ítems de la Escala de Satisfacción Familiar

\begin{tabular}{|c|c|c|c|c|c|}
\hline & \multicolumn{3}{|c|}{ Modelo bidimensional (M1) } & \multicolumn{2}{|c|}{ Modelo unidimensional (M2) } \\
\hline & $\mathrm{F}_{1}$ & $\mathrm{~F}_{2}$ & $h^{2}$ & $F_{1}$ & $\mathrm{~h}^{2}$ \\
\hline Ítem 1 & .786 & - & .618 & .779 & .607 \\
\hline Ítem 2 & - & .785 & .616 & .771 & .594 \\
\hline Ítem 3 & 696 & - & .484 & .706 & .498 \\
\hline Ítem 4 & - & .360 & .130 & .356 & .127 \\
\hline Ítem 5 & .562 & - & .316 & .519 & .269 \\
\hline Ítem 6 & - & .640 & .410 & .601 & .361 \\
\hline Ítem 7 & .640 & - & .410 & .636 & .404 \\
\hline Îtem 8 & - & .793 & .629 & .776 & .602 \\
\hline Ítem 9 & .436 & - & .190 & .433 & .187 \\
\hline Ítem 10 & - & .650 & .423 & .640 & .410 \\
\hline Ítem 11 & .690 & - & .476 & .686 & .471 \\
\hline Ítem 12 & - & .636 & .404 & .643 & .413 \\
\hline Ítem 13 & .800 & - & .640 & .794 & .630 \\
\hline Îtem 14 & .772 & - & .596 & .767 & .588 \\
\hline $\mathrm{F}_{1}$ & 1 & & & & \\
\hline $\mathrm{F}_{2}$ & .954 & 1 & & & \\
\hline AVE & .466 & .435 & - & .440 & - \\
\hline$\alpha$ Cronbach & .840 & .785 & - & .896 & - \\
\hline$\omega$ McDonald & .850 & .794 & - & 901 & - \\
\hline
\end{tabular}

Posteriormente, fue evaluado el modelo unidimensional, el cual presenta mejores índices de ajuste que el modelo anterior: $\chi^{2}{ }_{(74)}=164.635(p<.001) ; \chi^{2} /$ $\mathrm{gl}=2.225 ; \mathrm{CFI}=.995 ; \mathrm{GFI}=.990 ; \mathrm{AGFI}=.979 ; \mathrm{RMSEA}[\mathrm{IC} 90 \%]=.054$ [.032; .074]; $\mathrm{SRMR}=.060$. Aunque, al igual que el bidimensional, el promedio de varianza explicada de este modelo es menor de .5 $(\mathrm{AVE}=.440)$. Así mismo, la confiabilidad de este $(\alpha=.896 ; \omega=.901)$ es mayor que la del bifactorial, lo que nos lleva a concluir que la Escala de Satisfacción Familiar encaja mejor con una estructura unifactorial o unidimensional. 


\section{Discusión}

En este estudio se analizaron las propiedades psicométricas de la Escala de Satisfacción Familiar de Olson y Wilson (1982), que ha sido usada en el país en diversos estudios (Ferreira, 2003; Araujo, 2005; Sobrino, 2008; Capa et al., 2010; Mayorga \& Ñiquen, 2010). Igualmente, se han llevado a cabo varias investigaciones psicométricas de este instrumento (Bazo-Alvarez et al., 2016; Capa et al., 2010; Villarreal-Zegarra et al., 2017), destacándose los trabajos de Villarreal-Zegarra, que ha realizado investigaciones con las diferentes versiones del FACES.

Los resultados obtenidos en este trabajo son, por un lado, satisfactorios, pues la escala ha alcanzado puntajes adecuados de validez y confiabilidad. En el primer caso, se observa que los ítems pares e impares saturan en los factores de Adaptabilidad y Cohesión, salvo el ítem 14 que, en lugar de saturar en el factor de Adaptabilidad, lo hace en el de Cohesión (Tabla 2). Por otro lado, la confiabilidad obtenida mediante el alfa ordinal de cada uno de los factores es buena, registrando valores de .868 para la dimensión de Cohesión, y de .807, para la dimensión de Adaptabilidad.

Sin embargo, si bien la escala presenta adecuados valores de validez y confiabilidad tomando en cuenta el modelo original de dos factores, estos valores son más altos en el modelo unidimensional. En ese sentido, hay que hacer notar que ambos factores (Cohesión y Adaptabilidad) presentan una correlación muy alta $(\mathrm{r}=.954)$, lo que podría entenderse como una tendencia hacia la unidimensionalidad. Asimismo, luego de practicar el análisis factorial confirmatorio, los índices de bondad de ajuste son mejores para el modelo unidimensional que para el bifactorial, y la confiabilidad también se eleva a .911 con el alfa ordinal cuando el modelo es unidimensional.

Estos resultados son consistentes con lo reportado por el propio Olson (2014), que ha presentado una versión abreviada de dicha escala con solo 10 ítems y una estructura de un solo factor. En el Perú, un estudio reciente con 607 estudiantes universitarios ha adaptado dicha versión al español y ha realizado un análisis psicométrico que revela que la nueva versión de la Escala de Satisfacción Familiar presenta una solución unifactorial, además de brindar apoyo empírico con base en la estadística aplicada al modelo circumplejo de Olson (Villarreal-Zegarra et al., 2017).

Cabe recordar que, como indica Burga (2006), las soluciones unidimensionales son ideales en psicometría, pues ofrecen una versión más parsimoniosa de los constructos psicológicos; no obstante, dada la complejidad de los mismos por la diversidad de factores que los afectan (sociales, económicos, culturales, biológicos, psicológicos, etc.), los constructos psicológicos suelen constituirse en facto- 
res, componentes o dimensiones. Para el caso de la Escala de Satisfacción Familiar de Olson y Wilson, aunque hay estudios que validan su estructura bifactorial en muestras de jóvenes y/o adolescentes peruanos (Capa et al., 2010) o en trabajadores (Arias, Rivera \& Ceballos, 2017), otros, en cambio, revelan una tendencia hacia la unidimensionalidad, tal como sucede en la presente investigación o en la de Villarreal-Zegarra et al. (2017).

Este trabajo, entonces, contribuye al campo de la psicometría y de la psicología de la familia al presentar las propiedades psicométricas de la Escala de Satisfacción Familiar en una muestra de estudiantes universitarios de la ciudad de Arequipa, donde no hay estudios psicométricos previos sobre este instrumento, a pesar de que la familia ha sido y es uno de los fenómenos más investigados en esta región. En ese sentido, es importante contar con instrumentos que ofrezcan medidas de validez y confiabilidad adecuadas para las poblaciones de las diferentes regiones del país, ya que el Perú se caracteriza por su pluriculturalidad y su multietnicidad. Para ocasiones futuras, sería importante valorar otras propiedades psicométricas de la Escala de Satisfacción Familiar, como la invarianza o su validez concurrente. Además, deben tomarse muestras más representativas, de diversos estratos sociales, con rangos de edad más amplios y con estructuras familiares variadas, para hacer un procesamiento más completo y profundo de la data.

Queremos terminar enfatizando que la investigación en el campo de la psicología de la familia permitirá comprender los factores determinantes en la formación armoniosa y saludable de la persona humana (Melina, 2009), para lo cual, los estudios psicométricos de instrumentos especialmente diseñados para la valoración de los constructos relacionados con la familia son de importancia capital. Así, esperamos haber hecho una pequeña contribución para una mejor valoración de la familia a través de ajustes metodológicos más precisos de evaluación de la satisfacción familiar en la región Arequipa, y que sienta, a su vez, un antecedente relevante a nivel nacional.

\section{Referencias}

Aazami, S., Shamsuddin, K., \& Akmal, S. (2015). Malay version of the family satisfaction scale: Validity and reliability among malaysian working women. International Journal of Public Health Research, 5(2), 606-612.

Alarcón, R. (2014). Funcionamiento familiar y sus relaciones con la felicidad. Revista Peruana de Psicología y Trabajo Social, 3(1), 61-74.

Araujo, D. (2005). La satisfacción familiar y su relación con la agresividad y las estrategias de afrontamiento del estrés en adolescentes de Lima Metropolitana. Cultura, 19, 13-38. 
Araujo, D. (2007). Comunicación con los padres y factores de personalidad situacional en adolescentes de Educación Superior. Cultura, 21, 13-30.

Araujo, D. (2008). Comunicación padres-adolescente y estilos y estrategias de afrontamiento del estrés en escolares adolescentes de Lima. Cultura, 22, 227246.

Arias, W. L. (2012). Algunas consideraciones sobre la familia y la crianza desde un enfoque sistémico. Revista de Psicología de Arequipa, 2(1), 32-46.

Arias, W. L. (2013). Agresión y violencia en la adolescencia: La importancia de la familia. Avances en Psicología, 21(1), 23-34.

Arias, W. L., Castro, R., Dominguez, S., Masías, M., Canales, F., Castilla, S., \& Castilla, S. (2013). Construcción de un inventario de integración familiar. Avances en Psicología, 21(2), 195-206.

Arias, W. L., \& Ceballos, K. D. (2016). Síndrome de burnout, satisfacción laboral e integración familiar en trabajadores de una tienda por departamento de Arequipa. Illustro, 7, 43-58.

Arias, W. L., Galagarza, L. Y., Rivera, R., \& Ceballos, K. (2017). Análisis transgeneracional de la violencia familiar a través de la técnica de genogramas. Revista de Investigación en Psicología, 20(2), 283-308. doi: 10.15381/rinvp.v20i2.14042

Arias, W. L., Masías, M. A., Salas, X. S., Yépez, L., \& Justo, O. (2014). Integración familiar y felicidad en la ciudad de Arequipa. Revista de Psicología de Arequipa, 4(2), 204-215.

Arias, W. L., Quispe, A. C., \& Ceballos, K. D. (2016). Estructura familiar y nivel de logro en niños y niñas de escuelas públicas de Arequipa. Perspectiva de Familia, 1, 35-62.

Arias, W. L., Rivera, R., \& Ceballos, K. D. (2017). Análisis psicométrico de la Escala de Satisfacción Familiar de Wilson y Olson en una muestra de trabajadores de Arequipa. Ciencia E Trabajo. [Artículo sometido a revisión].

Bazo-Alvarez, J. C., Bazo-Alvarez, O. A., Aguila, J., Peralta, F., Mormontoy, W., \& Benett, I. M. (2016). Propiedades psicométricas de la Escala de Funcionalidad Familiar FACES-III: Un estudio en adolescentes peruanos. Revista Peruana de Medicina Experimental y Salud Pública, 33(3), 462-470. doi: 10.17843/rpmesp.2016.333.2299

Burga, A. (2006). La unidimensionalidad de un instrumento de medición: Perspectiva factorial. Revista de Psicología (Pontificia Universidad Católica del Perú), $24(1), 53-80$.

Byrne, B. (2010). Structural equation modeling with AMOS (2. ${ }^{\mathrm{a}}$ ed.). New York: Routledge, Taylor \& Francis Group. 
Capa, W., Vallejos, M., \& Cárdenas, R. (2010). Factores psicosociales asociados al consumo de drogas en adolescentes de una zona urbano popular de Lima Metropolitana. Revista de Investigaciones Psicológicas, 1, 21-37.

Cárdenas, M. V. (2016). Funcionamiento familiar, soporte social percibido y afrontamiento del estrés como factores asociados al bienestar psicológico en estudiantes de una universidad privada de Trujillo-La Libertad. Revista de Psicología [Universidad César Vallejo], 18(1), 72-85.

Castilla, H., Caycho, T., Shimabukuro, M., \& Valdivia, A. (2014). Percepción del funcionamiento familiar: Análisis psicométrico de la Escala APGAR-familiar en adolescentes de Lima. Propósitos y Representaciones, 2(1), 49-63. doi: 10.20511/pyr2014.v2.1.53

Castro, R., Arias, W. L., Dominguez, S., Masías, M. A., Salas, X., Canales, F., \& Flores, A. (2013). Integración familiar y variables socioeconómicas en Arequipa metropolitana. Revista de Investigación, 4, 35-65.

Castro, R., Cerellino, L. P., \& Rivera, R. (2017). Risk factors of violence against women in Peru. Journal of Family Violence, 32(8), 807-815. doi: 10.1007/s10896o17-9929-0

Castro, R., Riesco, G., \& Arela, R. (2016). ¿Familia y bienestar? Explorando la relación entre estructura familiar y satisfacción con la vida personal de las familias. Boletim Academia Paulista de Psicologia, 36(9o), 86-104.

Castro, R., \& Rivera, R. (2015). Mapa de la violencia contra la mujer: La importancia de la familia. Revista de Investigación, 6, 101-125.

Castro, R., Rivera, R., \& Seperak, R. (2017). Impacto de la composición familiar en los niveles de pobreza de Perú. Cultura Hombre Sociedad, 27(2), 69-88.

Caycho, T., Contreras, K., \& Merino, C. (2016). Percepción de los estilos de crianza y felicidad en adolescentes y jóvenes de Lima Metropolitana. Perspectiva de Familia, 1, 11-22.

Chuquimajo, S. (2017). Personalidad y clima social familiar en adolescentes de familia nuclear, biparental y monoparental. Revista de Investigación en Psicología, 20(2), 347-362. doi: 10.15381/rinvp.v20i2.14045

Copez-Lonzoy, A., Villarreal-Zegarra, D., \& Paz-Jesús, A. (2016). Propiedades psicométricas de la Escala de Comunicación Familiar en estudiantes universitarios. Revista Costarricense de Psicología, 35(1), 31-46.

Cruz, M. (2013). Clima social familiar y su relación con la madurez social del niño(a) de 6 a 9 años. Revista de Investigación en Psicología, 16(2), 157-179.

Delgado, P. (2016). Estrategias de negociación en parejas violentas y no violentas en Arequipa. Perspectiva de Familia, 1, 23-33. 
Dominguez, S., \& Alarcón, D. (2017). Análisis estructural de la Escala de Calidad de Interacción Familiar en escolares de Lima. Perspectiva de Familia, 2, 9-26.

Dominguez, S., Aravena, S., Ramírez, F., \& Yauri, C. (2013). Propiedades psicométricas de la Escala de Calidad de Interacción Familiar en escolares de Lima. Revista de Psicología [Universidad César Vallejo], 15(1), 55-77.

Ferreira, A. M. (2003). Sistema de interacción familiar asociado a la autoestima de menores en situación de abandono moral o prostitución. Revista de Investigación en Psicología, 6(2), 58-80.

Field, A. (2009). Discovering statistics using SPSS (3. - ed.). London: SAGE publications Ltd.

Fornell, C., \& Larcker, D. F. (1981). Evaluating structural equation models with unobservable variables and measurement error. Journal of Marketing Research, 18, 39-50. doi: 10.2307/3151312

Galagarza, L. Y., \& Arias, W. L. (2017). Alexitimia y funcionalidad familiar en estudiantes de ingeniería. Perspectiva de Familia, 2, 27-44.

García, C. B., \& Schneider, L. (2017). Abordaje sistémico en familias latinas sobrevivientes de violencia doméstica en Estados Unidos. Propósitos y Representaciones, 5(2), 277-298.

Gutara, L. (2016). Satisfacción familiar y resentimiento en estudiantes de secundaria de una institución pública de San Juan de Miraflores. Acta Psicológica Peruana, 1(1), 23-34.

Hae-Young, K. (2013). Statistical notes for clinical researchers: Assessing normal distribution (2) using skewness and kurtosis. Restorative Dentistry E Endodontics, 38(1), 52-54. doi: 10.5395/rde.2013.38.1.52

Hernández, R., Fernández, C., \& Baptista, P. (2006). Metodología de la investigación. México: McGraw-Hill.

JASP Team (2018). JASP (version o.10.0) [programa informático].

Jöreskog, K. (1969). A general approach to confirmatory maximun likelihood factor analysis. Psychometrika, 34, 183-202.

Laguna, J. P., \& Rodríguez, A. S. (2008). Comportamientos socioemocionales de resiliencia en preescolares procedentes de hogares mono y biparentales. Revista de Psicología [Universidad Católica de Santa María], 5, 52-65.

Lee, S. Y., Poon, W. Y., \& Bentler, P. M. (1995). A two-stage estimation of structural equation models with continuous and polytomous variables. British Journal of Mathematical and Statistical Psychology, 48(2), 339-358. doi: 10.1007/BFo2294660

Malgady, R. (2007). How skew are psychological data? A standardized index of effect size. The Journal of General Psychology, 134(3), 355-359. doi: 10.3200/GENP.134.3.355-360 
Mallma, N. (2016). Relaciones intrafamiliares de dependencia emocional en estudiantes de psicología de un centro de formación superior. Acta Psicológica Peruana, 1(1), 107-124.

Manrique, D. L., Ghesquière, P., \& Leeuwen, K. van. (2014). Evaluation of Parental Behavior Scale in Peruvian Context. Journal of Children and Family Studies, 23(5), 885-894. doi: 10.1007/s10826-013-9744-z

Matalinares, M., Arenas, C., Sotelo, L., Díaz, G., Dioses, A., Yaringaño, J.... Tipacti, R. (2010). Clima familiar y agresividad en estudiantes de secundaria de Lima Metropolitana. Revista de Investigación en Psicología, 13(1), 109-128.

Matalinares, M., Raymundo, O., \& Baca, D. (2014). Propiedades psicométricas de la escala de estilos Parentales. Persona, 17, 95-121.

Mayorga, E., \& Ñiquen, M. (2010). Satisfacción familiar y expresión de la cólera-hostilidad en adolescentes escolares que presentan conductas antisociales. Revista de Investigaciones Psicológicas, 1, 87-92.

Melina, L. (2009). Por una cultura de la familia. Valencia: Edicep.

Merino, C., \& Arndt, S. (2004). Análisis factorial confirmatorio de la Escala de Estilos de Crianza de Steinberg: Validez preliminar de constructo. Revista de Psicología [Pontificia Universidad Católica del Perú], 22(2), 187-214.

Merino, C., Díaz, M., \& Cohen, B. H. (2003). De los niños a los padres: El inventario de percepción de conductas parentales. Persona, 6, 135-149.

Merino, C., Díaz, M., \& DeRoma, V. (2004). Validación del instrumento de conductas parentales: Un análisis factorial confirmatorio. Persona, 7, 145-162.

Miljánovich, M. A., Huerta, R. E., Campos, E., Torres, S., Vásquez, V. A., Vera, K., \& Díaz, A. (2013). Violencia familiar: modelos explicativos del proceso a través del estudio de casos. Revista de Investigación en Psicología, 16(1), 29-44.

Miljánovich, M. A., Nolberto, V., Martina, M., Huerta, R. E., Torres, S., \& Camones, F. (2010). Perú: Mapa de violencia familiar, a nivel departamental, según la ENDES 2007-2008. Características e implicancias. Revista de Investigación en Psicología, 13(2), 191-205.

Minetto, A. B. (2013). Violencia familiar e ideación suicida en adolescentes del Hospital San Juan Bautista de Huaral. PsiqueMag, 2(1), 63-76.

Montero, I., \& León, O. (2002). Clasificación y descripción de las metodologías de investigación en Psicología. Revista Internacional de Psicología Clínica y de la Salud, 2(3), 503-508.

Muñoz, Z. E. (2016). Estilos de socialización parental y dependencia emocional en mujeres de 16 y 17 años de edad en instituciones educativas nacionales de Lima, 2014. PsiqueMag, 4(1), 81-101.

Murillo, M. G. (2015). Desarrollo del juicio moral en personas casadas y divorciadas en la ciudad de Arequipa. Avances en Psicología, 23(1), 73-85. 
Muthén, B. O., Toit, S. H. C. du, \& Spisic, D. (1997). Robust inference using weighted least squares and quadratic estimating equations in latent variable modeling with categorical and continuous outcomes [artículo en línea]. Recuperado el 15 de octubre de 2016 de: http://www.statmodel.com/bmuthen/articles/ Article_075.pdf

Olson, D. H. (1986). Circumplex model VII: Validation studies and FACES III. Family Process Journal, 25, 337-351.

Olson, D. H. (1993). Circumplex model of family systems: Assessing functioning. En Walsh, F. (Ed.). Normal family processes (pp. 73-103). New York: Guilford.

Olson, D. H. (2000). Circumplex model of marital and family systems. Journal of Family Therapy, 22, 144-167.

Olson, D. H. (2014). Family Satisfaction Scale. Minnesota: Life Innovations, Inc.

Olson, D. H., Portner, J., \& Lavee, Y. (1985). FACES III. St. Paul: University of Minnesota, Department of Family Science.

Olson, D. H., Russell, C., \& Sprenkle, D. H. (1989). Circumplex model of marital and family systems II: Empirical studies and clinical intervention. Advances in Family Intervention, Assessment and Theory, 1, 129-176.

Olson, D. H., \& Wilson, L. R. (1982). Family Satisfaction Scale. En Olson, D. H., McCubbin, H. I., Barnes, H., Larsen, A., Muxen, M., \& Wilson, M. (Eds.). Family inventories: Inventories used in a national survey of families across the family life cycle (pp. 43-49). St. Paul, MN: University of Minnesota.

Oporto, C., \& Zanabria, L. (2006). Inteligencia emocional en hijos de familias nucleares y monoparentales. Revista de Psicología [Universidad Católica de Santa María], 3, 25-36.

Oruna, A. (2016). Ambiente familiar y percepción de la autoeficacia en estudiantes de ciencias de la salud de una universidad privada de Huacho. Acta Psicológica Peruana, 1(2), 325-352.

Pérez, P. Z. (2016). Funcionamiento familiar e ideación suicida en alumnos de $5 .^{\circ}$ año de educación secundaria del distrito de San Juan de Miraflores. PsiqueMag, 4(1), 81-93.

Pliego, F., \& Castro, R. (2015). Tipos de familia y bienestar de niños y adultos. El debate cultural del siglo XXI en 13 países democráticos. Arequipa: Universidad Católica San Pablo.

Prado, T. R., \& Águila, M. del. (2004). Estructura y funcionamiento familiar en adolescentes resilientes. Teoría e Investigación en Psicología, 13, 85-113.

Prado, T. R., \& Águila, M. del. (2010). Ajuste y satisfacción en parejas que trabajan. Revista de Investigaciones Psicológicas, 1(1), 38-52. 
Rebaza, R. P., \& Julca, M. B. (2009). Satisfacción marital y ansiedad por concebir un hijo en mujeres con diagnóstico de infertilidad. Revista de Psicología [Universidad César Vallejo], 11, 79-96.

$\mathrm{R}$ Core Team (2017). R: A language and environment for statistical computing. $\mathrm{R}$ Foundation for Statistical Computing, Vienna, Austria. Recuperado el 23 de marzo de 2018 de: https://www.R-project.org/

Reusche, R. M. (1995). Estructura y funcionamiento familiar en un grupo de estudiantes de secundaria de nivel socioeconómico medio con alto y bajo rendimiento escolar. Avances en Psicología, 3, 163-19o.

Reusche, R. M. (1999). El afecto y la autoridad familiar en adolescentes. Revista Peruana de Psicología, 4(7-8), 193-182.

Riesco, R., \& Arela, R. (2015). Impacto de la estructura familiar en la satisfacción con los ingresos en los hogares urbanos en Perú. Economía, 38(76), 51-76.

Rivera, R., Arias, W. L., \& Cahuana, M. [Artículo en revisión]. Perfil familiar de adolescentes con sintomatología depresiva en la ciudad de Arequipa, Perú. Revista Chilena de Neuropsiquiatría.

Rivera, R., \& Cahuana, M. (2016). Influencia de la familia sobre las conductas antisociales en adolescentes de Arequipa-Perú. Actualidades en Psicología, 30(120), 85-97. doi: 10.15517/ap.v30i120.18814

Rosas, B. (2014). Percepción de los vínculos parentales y funcionamiento familiar en sujetos drogodependientes. Un recurso a explorar en el proceso de rehabilitación. PsiqueMag, 3(1), 81-101.

Rossel, Y. (2012). lavaan: An R Package for Structural Equation Modeling. Journal of Statistical Software, 48(2), 1-36.

Salvatierra, B. (1992). ¿Qué hago? Arequipa: UNSA.

Salvatierra, B. (1993). El matrimonio. Arequipa: UNSA.

Salvatierra, B. (1997). Orientación psicológica para la familia. Arequipa: UNSA.

Schmidt, V., Barreyro, J. P., \& Maglio, A. L. (2009). Escala de evaluación del funcionamiento familiar FACES III: ¿Modelo de dos o tres factores? Escritos de Psicología, 3(2), 30-6.

Seperak, R. (2016). Motivos intrínsecos, extrínsecos y trascendentes en la decisión de contraer matrimonio en casados, separados y/o divorciados. Revista de Psicología de Arequipa, 6(1), 349-368.

Silva, C., \& Argote, C. (2007). Actitudes hacia matrimonio y divorcio en jóvenes procedentes de familias intactas y divididas. Revista de Psicología [Universidad Católica de Santa María], 4, 29-37.

Sobrino, L. (1999). Terapia estratégica. Revista Peruana de Psicología, 4(7-8), 51-62. 
Sobrino, L. (2008). Niveles de satisfacción familiar y de comunicación entre padres e hijos. Avances en Psicología, 16(1), 109-137.

Sotil, A. (2002). Influencia del clima familiar: Estrategias de aprendizaje e inteligencia emocional en el rendimiento académico. Revista de Investigación en Psicología, 5(1), 53-69.

Tercero, G., Lavielle, M. P. D., Muñoz, O., Clark, P., Medeiros, M., Hernández, A., \& Luque, M. (2013). Escala de Satisfacción Familiar por Adjetivos (ESFA) en escolares y adolescentes mexicanos: Datos normativos. Salud Mental, 36(5), 381-386.

Tirado, P., Álvarez, V., Chávez, M., Holguín, S., Honorio, A., Moreno, M.... Uribe, M. (2008). Satisfacción familiar y salud mental en alumnos universitarios ingresantes. Revista de Psicología [Universidad César Vallejo], 10, 42-48.

Uderhill, A. T., LoBello, S. G., \& Fine, P. R. (2004). Reliability and validity of the family satisfaction Scale with survivors of traumatic brain injury. Journal of Rehabilitation Research E Development, 41(4), 603-610.

Vidal, G. (2014). Del proyecto a la acción: Historia de la creación y labor del Instituto de la Familia de la UNIFÉ. Persona y Familia, 3, 83-105.

Villarreal-Zegarra, D. (2015). Funcionalidad familiar y composición familiar en adolescentes de quinto año de secundaria. Revista Peruana de Psicología y Trabajo Social, 4(1), 37-46.

Villarreal-Zegarra, D., \& Paz-Jesús, A. (2015). Terapia familiar sistémica: Una aproximación a la teoría y la práctica clínica. Interacciones. Revista de Avances en Psicología, 1(1), 45-55.

Villarreal-Zegarra, D., \& Paz-Jesús, A. (2017). Cohesión, adaptabilidad y composición familiar en adolescentes del Callao, Perú. Propósitos y Representaciones, 5(2), 21-42.

Villarreal-Zegarra, D., Paz-Jesús, A., Copez-Lonzoy, A., \& Costa-Ball, C. D. (2017). Validez y confiabilidad de la Escala de Satisfacción Familiar en estudiantes universitarios de Lima Metropolitana, Perú. Actualidades en Psicología, 31(123), 89-98. doi: 10.15517/ap.v31i123.23573

Yucra, J. (2016). Funcionamiento familiar y habilidades sociales como factores asociados a desórdenes emocionales en universitarios. Acta Psicológica Peruana, 1(1), 11-22. 